\title{
G-Metric spaces in any number of arguments and related fixed-point theorems
}

\author{
Antonio Roldán ${ }^{1 *}$, Erdal Karapınar² and Poom Kumam³
}

${ }^{\text {"Correspondence: }}$

afroldan@ujaen.es

1 University of Jaén, Campus las Lagunillas s/n, Jaén, 23071, Spain

Full list of author information is

available at the end of the article

\begin{abstract}
Inspired by the notion of Mustafa and Sims' G-metric space and the attention that this kind of metric has received in recent times, we introduce the concept of a $G$-metric space in any number of variables, and we study some of the basic properties. Then we prove that the family of this kind of metric is closed under finite products. Finally, we show some fixed-point theorems that improve and extend some well-known results in this field.
\end{abstract}

MSC: 46T99; 47H10;47H09; 54H25

Keywords: partially ordered set; fixed point; contractive mapping; G-metric space

\section{Introduction}

In the 1960s, Gähler [1,2] tried to generalize the notion of metric and introduced the concept of 2-metric spaces inspired by the mapping that associated the area of a triangle to its three vertices. Later, Dhage [3] changed the axioms and presented the concept of a D-metric. Unfortunately, both kinds of metrics appear not to have as good properties as their authors announced (see [4-10]). To overcome these drawbacks, Mustafa and Sims [11] presented the notion of a G-metric space, which have received much attention since then. The literature on this topic, especially in related fixed point theory, has grown a lot in recent times (see, for instance, [12-26] and references therein).

The main aim of the present paper is to introduce the notion of a G-metric space in any number of variables. To do that, we have been inspired by the perimeter of a triangle, as well as Dhage, which in the multidimensional case can be seen as the sum of all distances between any pair of points. In this sense, the axioms we present and the properties we deduce are very natural. We also prove two relevant facts: the product of metrics of this kind is also a metric in this sense, and there is no a trivially way to reduce the number of variables (which, for instance, permits us to reduce a $G_{n}^{*}$-metric to the Mustafa and Sims' spaces). Later, we demonstrate some fixed-point theorems distinguishing between the axioms that the metric verifies $\left(G_{n}^{*}\right.$-metrics and $G_{n}$-metrics). As a consequence, our main results are, obviously, valid in the context of $G$-metric spaces.

\section{Preliminaries}

Let $n$ be a positive integer such that $n \geq 2$. Henceforth, $X$ will denote a non-empty set and $X^{n}$ will denote the product space $X \times X \times \stackrel{n}{\cdots} \times X$. Throughout this manuscript, $m$ and $k$ will denote non-negative integers. Unless otherwise stated, 'for all $m$ ' will mean 'for all $m \geq 0$. Let $\mathbb{R}_{0}^{+}=[0, \infty)$ and let $\mathbb{N}=\{0,1,2, \ldots\}$.

@ 2014 Roldán et al.; licensee Springer. This is an Open Access article distributed under the terms of the Creative Commons Attribution License (http://creativecommons.org/licenses/by/2.0), which permits unrestricted use, distribution, and reproduction in any medium, provided the original work is properly cited. 
Proposition 1 If $\left\{a_{m}^{1}\right\}_{m \geq 0},\left\{a_{m}^{2}\right\}_{m \geq 0}, \ldots,\left\{a_{m}^{N}\right\}_{m \geq 0} \subset \mathbb{R}_{0}^{+}$are $N$ sequences of non-negative real numbers and we define $b_{m}=\max \left(a_{m}^{1}, a_{m}^{2}, \ldots, a_{m}^{N}\right)$ for all $m \geq 0$, then $\left\{b_{m}\right\}_{m \geq 0} \rightarrow 0$ if, and only if, $\left\{a_{m}^{j}\right\}_{m \geq 0} \rightarrow 0$ for all $j \in\{1,2, \ldots, N\}$.

Definition 2 (Khan et al. [27]) An altering distance function is a continuous, nondecreasing mapping $\phi: \mathbb{R}_{0}^{+} \rightarrow \mathbb{R}_{0}^{+}$such that $\phi^{-1}(\{0\})=\{0\}$. Let $\Psi$ denote the family of all altering distance functions.

Lemma 3 If $\psi \in \Psi$ and $\left\{a_{m}\right\} \subset[0, \infty)$ verifies $\left\{\psi\left(a_{m}\right)\right\} \rightarrow 0$, then $\left\{a_{m}\right\} \rightarrow 0$.

Corollary 4 If $\psi, \varphi \in \Psi$ and $\left\{a_{m}\right\} \subset[0, \infty)$ verifies $\psi\left(a_{m+1}\right) \leq(\psi-\varphi)\left(a_{m}\right)$ for all $m$, then $\left\{a_{m}\right\} \rightarrow 0$.

The following kind of mapping was introduced by Popescu in [28] and Moradi and Farajzadeh in [29].

Definition 5 We will denote by $\Phi$ the family of all mappings $\varphi: \mathbb{R}_{0}^{+} \rightarrow \mathbb{R}_{0}^{+}$verifying:

$$
\text { if }\left\{t_{m}\right\}_{m \in \mathbb{N}} \subset \mathbb{R}_{0}^{+} \text {and }\left\{\varphi\left(t_{m}\right)\right\} \rightarrow 0 \text {, then }\left\{t_{m}\right\} \rightarrow 0 \text {. }
$$

Remark 6 Obviously, $\Psi \subset \Phi$.

Definition 7 We will say that $\preccurlyeq$ is a partial preorder on $X$ (or $(X, \preccurlyeq)$ is a preordered set or $(X, \preccurlyeq)$ is a partially preordered space) if the following properties hold.

- Reflexivity: $x \preccurlyeq x$ for all $x \in X$.

- Transitivity: If $x, y, z \in X$ verify $x \preccurlyeq y$ and $y \preccurlyeq z$, then $x \preccurlyeq z$.

Definition 8 (Mustafa and Sims [11]) A generalized metric (or a G-metric) on $X$ is a mapping $G: X^{3} \rightarrow \mathbb{R}_{0}^{+}$verifying, for all $x, y, z \in X$ :

$\left(\mathrm{G}_{1}\right) \quad G(x, x, x)=0$.

$\left(\mathrm{G}_{2}\right) \quad G(x, x, y)>0$ if $x \neq y$.

$\left(\mathrm{G}_{3}\right) \quad G(x, x, y) \leq G(x, y, z)$ if $y \neq z$.

$\left(\mathrm{G}_{4}\right) G(x, y, z)=G(x, z, y)=G(y, z, x)=\cdots$ (symmetry in all three variables).

$\left(\mathrm{G}_{5}\right) G(x, y, z) \leq G(x, a, a)+G(a, y, z)$ (rectangle inequality).

In [11], the authors proved that, in general, the product space of G-metric spaces is not a $G$-metric space (unless the factors are symmetric, that is, that they can be reduced to metric spaces). To overcome this drawback, Roldán and Karapınar introduced the concept of $G^{*}$-metric spaces, in which the axiom $\left(G_{3}\right)$ is omitted.

Definition 9 (Roldán and Karapınar [20]) A $G^{*}$-metric on $X$ is a mapping $G: X^{3} \rightarrow \mathbb{R}_{0}^{+}$ verifying $\left(G_{1}\right),\left(G_{2}\right),\left(G_{4}\right)$ and $\left(G_{5}\right)$.

Using this class of spaces, these authors proved that the product of $G^{*}$-metric spaces is also a $G^{*}$-metric space, and they also showed some related fixed point results. This is the case of the generalized metrics that we are going to introduce in the following section. 


\section{Multidimensional $G^{*}$-metric spaces}

The following definition is a natural extension of the concept of a G-metric space. Note that, for convenience, we change the order in which we present the axioms with respect to the order Mustafa and Sims chose.

Definition 10 A $G_{n}^{*}$-metric on $X$ is a mapping $\mathcal{G}: X^{n} \rightarrow \mathbb{R}_{0}^{+}$verifying, for all $x_{1}, x_{2}, \ldots, x_{n}$, $x, y, a \in X$ :

$\left(\mathrm{A}_{1}\right) \mathcal{G}(x, x, \ldots, x)=0$.

$\left(\mathrm{A}_{2}\right)$ If $x_{1} \neq x_{2}$ then $\mathcal{G}\left(x_{1}, x_{2}, x_{3}, \ldots, x_{n}\right)>0$.

$\left(\mathrm{A}_{3}\right)$ If $\sigma: \Lambda_{n} \rightarrow \Lambda_{n}$ is a permutation, then $\mathcal{G}\left(x_{\sigma(1)}, x_{\sigma(2)}, \ldots, x_{\sigma(n)}\right)=\mathcal{G}\left(x_{1}, x_{2}, \ldots, x_{n}\right)$ (symmetry in all its variables).

$\left(\mathrm{A}_{4}\right) \mathcal{G}\left(x_{1}, x_{2}, x_{3}, \ldots, x_{n}\right) \leq \mathcal{G}\left(x_{1}, a, a, a, \ldots, a\right)+\mathcal{G}\left(a, x_{2}, x_{3}, x_{4}, \ldots, x_{n}\right)$ (multidimensional inequality).

We will say that $\mathcal{G}$ is a $G_{n}$-metric (or a $G$-metric on $n$ variables) if it also verifies ${ }^{\text {a }}$ :

$\left(\mathrm{A}_{5}\right)$ If $x \neq y \neq x_{3} \neq x_{4} \neq \cdots \neq x_{n-1} \neq x_{n}$, then $\mathcal{G}(x, x, x, \ldots, x, y) \leq \mathcal{G}\left(x, y, x_{3}, x_{4}, \ldots, x_{n}\right)$.

Remark 11 Following the previous definition, it is not difficult to prove that a $G_{2}^{*}$-metric space is a classical metric space, a $G_{3}$-metric space if a $G$-metric space in the sense of Mustafa and Sims [11], and a $G_{3}^{*}$-metric space if a $G^{*}$-metric space in the sense of Roldán and Karapinar [20].

However, a $G_{n}^{*}$-metric does not generate a $G_{n-1}^{*}$-metric in a trivial way since, if $\mathcal{G}$ is a $G_{n}^{*}$-metric on $X$ and we define $\mathcal{G}_{1}, \mathcal{G}_{2}: X^{n-1} \rightarrow \mathbb{R}_{0}^{+}$, for all $\left(x_{1}, x_{2}, \ldots, x_{n-1}\right) \in X^{n-1}$, by

$$
\begin{aligned}
& \mathcal{G}_{1}\left(x_{1}, x_{2}, \ldots, x_{n-1}\right)=\mathcal{G}\left(x_{1}, x_{1}, x_{2}, x_{3}, \ldots, x_{n-1}\right) \text { and } \\
& \mathcal{G}_{2}\left(x_{1}, x_{2}, \ldots, x_{n-1}\right)=\mathcal{G}\left(z_{0}, x_{1}, x_{2}, x_{3}, \ldots, x_{n-1}\right)
\end{aligned}
$$

(where $z_{0} \in X$ is fixed), then $\mathcal{G}_{1}$ and $\mathcal{G}_{2}$ are not $G_{n-1}^{*}$-metrics ( $\mathcal{G}_{1}$ does not have to verify $\left(\mathrm{A}_{3}\right)$ and $\mathcal{G}_{2}$ need not verify $\left.\left(\mathrm{A}_{1}\right)\right)$.

Example 12 Each metric space $(X, d)$ can be provided with a $G_{n}$-metric defining $\mathcal{G}_{d}$ : $X^{n} \rightarrow \mathbb{R}_{0}^{+}$by

$$
\mathcal{G}_{d}\left(x_{1}, x_{2}, \ldots, x_{n}\right)=\frac{1}{2} \sum_{i, j=1}^{n} d\left(x_{i}, x_{j}\right)=\sum_{i, j=1, i<j}^{n} d\left(x_{i}, x_{j}\right) \quad \text { for all }\left(x_{1}, x_{2}, \ldots, x_{n}\right) \in X^{n} .
$$

Lemma 13 If $(X, \mathcal{G})$ is a $G_{n}^{*}$-metric space and we define $d_{\mathcal{G}}: X \times X \rightarrow \mathbb{R}_{0}^{+}$by

$$
d_{\mathcal{G}}(x, y)=\max (\mathcal{G}(x, y, y, \ldots, y), \mathcal{G}(y, x, x, \ldots, x)) \text { for all } x, y \in X,
$$

then $d_{\mathcal{G}}$ is a metric on $X$. Furthermore, if $d$ is a metric on $X$, then $d_{\mathcal{G}_{d}}=(n-1) d$.

For brevity, when the last arguments are repeated, we will denote

$$
\begin{aligned}
& \mathcal{G}(x, y, y, y, \ldots, y) \quad \text { by } \mathcal{G}\left(x,[y]^{n-1}\right) \quad \text { and } \\
& \mathcal{G}(x, y, z, z, z, \ldots, z) \quad \text { by } \mathcal{G}\left(x, y,[z]^{n-2}\right) \text { or } \mathcal{G}\left(x,[z]^{n-2}, y\right) .
\end{aligned}
$$


Corollary 14 If $(X, \mathcal{G})$ is a $G_{n}^{*}$-metric space and we define $d_{\mathcal{G}}^{s}: X \times X \rightarrow \mathbb{R}_{0}^{+}$by

$$
d_{\mathcal{G}}^{s}(x, y)=\mathcal{G}\left(x,[y]^{n-1}\right)+\mathcal{G}\left(y,[x]^{n-1}\right) \quad \text { for all } x, y \in X,
$$

then $d_{\mathcal{G}}^{s}$ is also a metric on $X$. Moreover, $d_{\mathcal{G}} \leq d_{\mathcal{G}}^{s} \leq 2 d_{\mathcal{G}}$.

The metric $d_{\mathcal{G}}$ generates a unique Hausdorff topology $\tau_{\mathcal{G}}$ on each $G_{n}^{*}$-metric space $(X, \mathcal{G})$ such that $\beta_{x}^{\mathcal{G}}=\left\{B_{\mathcal{G}}(x, r): r>0\right\}$ is a neighborhood system at each $x \in X$, where $B_{\mathcal{G}}(x, r)$ denotes the ball $\left\{y \in X: \max \left(\mathcal{G}\left(x,[y]^{n-1}\right), \mathcal{G}\left(y,[x]^{n-1}\right)\right)<r\right\}$. This topology yields the following notions of convergence, Cauchy sequence, completeness, and continuity.

Definition 15 Let $(X, \mathcal{G})$ be a $G_{n}^{*}$-metric space, let $\left\{x_{m}\right\} \subseteq X$ be a sequence and let $x \in X$ be a point. We will say that:

- $\left\{x_{m}\right\} \mathcal{G}$-converges to $x$ (we will denote this by $\left\{x_{m}\right\} \stackrel{\mathcal{G}}{\rightarrow} x$ ) if

$$
\lim _{m_{1}, m_{2}, \ldots, m_{n-1} \rightarrow \infty} \mathcal{G}\left(x_{m_{1}}, x_{m_{2}}, \ldots, x_{m_{n-1}}, x\right)=0
$$

that is, for all $\varepsilon>0$ there is $m_{0} \in \mathbb{N}$ such that if $m_{1}, m_{2}, \ldots, m_{n-1} \geq m_{0}$, then $\mathcal{G}\left(x_{m_{1}}, x_{m_{2}}, \ldots, x_{m_{n-1}}, x\right)<\varepsilon$;

- $\left\{x_{m}\right\}$ is a $\mathcal{G}$-Cauchy sequence if $\lim _{m_{1}, m_{2}, \ldots, m_{n} \rightarrow \infty} \mathcal{G}\left(x_{m_{1}}, x_{m_{2}}, \ldots, x_{m_{n-1}}, x_{m_{n}}\right)=0$ (that is, for all $\varepsilon>0$ there is $m_{0} \in \mathbb{N}$ such that if $m_{1}, m_{2}, \ldots, m_{n-1}, m_{n} \geq m_{0}$, then $\left.\mathcal{G}\left(x_{m_{1}}, x_{m_{2}}, \ldots, x_{m_{n-1}}, x_{m_{n}}\right)<\varepsilon\right)$

- a subset $A \subseteq X$ is $\mathcal{G}$-complete is every $\mathcal{G}$-Cauchy sequence in $A$ is $\mathcal{G}$-convergent in $A$;

- a mapping $F: X^{N} \rightarrow X$ is $\mathcal{G}$-continuous if for all $N$ sequences $\left\{x_{m}^{1}\right\},\left\{x_{m}^{2}\right\}, \ldots,\left\{x_{m}^{N}\right\} \subseteq X$ such that $\left\{x_{m}^{i}\right\} \stackrel{\mathcal{G}}{\rightarrow} z^{i} \in X$ for all $i \in\{1,2, \ldots, N\}$, we have $\left\{F\left(x_{m}^{1}, x_{m}^{2}, \ldots, x_{m}^{N}\right)\right\} \stackrel{\mathcal{G}}{\rightarrow} F\left(z^{1}, z^{2}, \ldots, z^{N}\right)$.

Notice that, by the symmetry condition $\left(\mathrm{A}_{3}\right)$, we could reduce the previous definitions to the case in which $m_{n} \geq m_{n-1} \geq \cdots \geq m_{2} \geq m_{1} \geq m_{0}$. When the $G_{n}^{*}$-metric space is preordered, we can also consider the following class of spaces.

Definition 16 Let $(X, \mathcal{G})$ be a $G_{n}^{*}$-metric space and let $\preccurlyeq$ be a preorder on $X$. We will say that $(X, \mathcal{G}, \preccurlyeq)$ is regular-non-decreasing if it verifies the following property:

$\triangleright$ If $\left\{x_{m}\right\} \subseteq X$ is a $\preccurlyeq$-non-decreasing sequence $\left(x_{m} \preccurlyeq x_{m+1}\right.$ for all $m$ ) that $\mathcal{G}$-converges to $x \in X$, then $x_{m} \preccurlyeq x$ for all $m$.

We will say that $(X, \mathcal{G}, \preccurlyeq)$ is regular-non-increasing when:

$\triangleright$ If $\left\{y_{m}\right\} \subseteq X$ is a $\preccurlyeq$-non-increasing sequence $\left(y_{m} \succcurlyeq y_{m+1}\right.$ for all $\left.m\right)$ that $\mathcal{G}$-converges to $y \in X$, then $y_{m} \succcurlyeq y$ for all $m$.

The space $(X, \mathcal{G}, \preccurlyeq)$ is regular if it is both regular-non-decreasing and regular-nonincreasing.

Some properties of a $G_{n}^{*}$-metric space are listed in the following result.

Lemma 17 Let $(X, \mathcal{G})$ be a $G_{n}^{*}$-metric space, let $\left\{x_{m}\right\} \subseteq X$ be a sequence and let $x, y, x_{1}, x_{2}, x_{3}$, $\ldots, x_{n} \in X$. 
(1) If there exist $i, j \in\{1,2, \ldots, n\}$ such that $x_{i} \neq x_{j}$, then $\mathcal{G}\left(x_{1}, x_{2}, x_{3}, \ldots, x_{n}\right)>0$.

(2) If $\mathcal{G}\left(x_{1}, x_{2}, x_{3}, \ldots, x_{n}\right)=0$, then $x_{1}=x_{2}=x_{3}=\cdots=x_{n}$.

(3) $\mathcal{G}\left(x,[y]^{n-1}\right) \leq(n-1) \mathcal{G}\left(y,[x]^{n-1}\right)$.

(4) $\mathcal{G}\left(x_{1}, x_{2}, x_{3}, \ldots, x_{n}\right) \leq \sum_{i=1}^{n} \mathcal{G}\left(x_{i},[a]^{n-1}\right)$ for all $a \in X$.

(5) $\left\{x_{m}\right\} \mathcal{G}$-converges to $x$ if, and only if, $\lim _{m \rightarrow \infty} \mathcal{G}\left(x_{m},[x]^{n-1}\right)=0$, which is equivalent to $\left\{x_{m}\right\} d_{\mathcal{G}}$-converges to $x$.

(6) $\left\{x_{m}\right\}$ is $\mathcal{G}$-Cauchy if, and only if, it is $d_{\mathcal{G}}$-Cauchy.

Proof (1) Suppose that $x_{i} \neq x_{j}(i \neq j)$ and let $\sigma: \Lambda_{n} \rightarrow \Lambda_{n}$ be any permutation such that $\sigma(1)=i$ and $\sigma(2)=j$. Then, by axiom $\left(\mathrm{A}_{2}\right)$

$$
\mathcal{G}\left(x_{1}, x_{2}, x_{3}, \ldots, x_{n}\right)=\mathcal{G}\left(x_{\sigma(1)}, x_{\sigma(2)}, x_{\sigma(3)}, \ldots, x_{\sigma(n)}\right)=\mathcal{G}\left(x_{i}, x_{j}, x_{\sigma(3)}, \ldots, x_{\sigma(n)}\right)>0
$$

(2) The first item establishes that if two points are different, then the $\mathcal{G}$-metric is strictly positive. Then, if the $\mathcal{G}$-metric takes the value zero, then all points must be equal.

(3) Taking into account that $\mathcal{G}$ is symmetric in all its variables, we can apply $n-1$ times the axiom $\left(\mathrm{A}_{4}\right)$ using $a=x$ to deduce

$$
\begin{aligned}
\mathcal{G}(x, y, y, y, \ldots, y) & =\mathcal{G}(\underbrace{y, y, y, \ldots, y, y}_{n-1}, x) \leq \mathcal{G}(y, \underbrace{x, x, x, \ldots, x, x}_{n-1})+\mathcal{G}(x, \underbrace{y, y, y, \ldots, y, y}_{n-2}, x) \\
& =\mathcal{G}(y, x, x, x, \ldots, x, x)+\mathcal{G}(\underbrace{y, y, y, \ldots, y, y}_{n-2}, x, x) \\
& \leq \mathcal{G}(y, x, x, x, \ldots, x, x)+(\mathcal{G}(y, x, x, x, \ldots, x, x)+\mathcal{G}(x, y, y, y, \ldots, y, y, x, x) \\
& =2 \mathcal{G}(y, x, x, x, \ldots, x, x)+\mathcal{G}(\underbrace{y, y, y, \ldots, y, y}_{n-3}, x, x, x) \\
& \leq \ldots \leq(n-1) \mathcal{G}(y, x, x, x, \ldots, x, x) .
\end{aligned}
$$

(4) By $\left(\mathrm{A}_{4}\right)$ and $\left(\mathrm{A}_{3}\right)$,

$$
\begin{aligned}
\mathcal{G}\left(x_{1}, x_{2}, x_{3}, \ldots, x_{n}\right) & \leq \mathcal{G}\left(x_{1},[a]^{n-1}\right)+\mathcal{G}\left(a, x_{2}, x_{3}, \ldots, x_{n}\right) \\
& =\mathcal{G}\left(x_{1},[a]^{n-1}\right)+\mathcal{G}\left(x_{2}, x_{3}, x_{4}, \ldots, x_{n}, a\right) \\
& \leq \mathcal{G}\left(x_{1},[a]^{n-1}\right)+\mathcal{G}\left(x_{2}, a, a, \ldots, a, a\right)+\mathcal{G}\left(a, x_{3}, x_{4}, \ldots, x_{n}, a\right) \\
& =\mathcal{G}\left(x_{1},[a]^{n-1}\right)+\mathcal{G}\left(x_{2},[a]^{n-1}\right)+\mathcal{G}\left(x_{3}, x_{4}, \ldots, x_{n}, a, a\right) \leq \ldots \\
& \leq \sum_{i=1}^{n} \mathcal{G}\left(x_{i},[a]^{n-1}\right) .
\end{aligned}
$$

(5) Suppose that $\left\{x_{m}\right\} \mathcal{G}$-converges to $x$, and let $\varepsilon>0$ be arbitrary. Using $\varepsilon^{\prime}=\varepsilon /(n-$ 1) $>0$, by hypothesis, there exists $m_{0} \in \mathbb{N}$ such that if $m_{1}, m_{2}, \ldots, m_{n-1} \geq m_{0}$, then $\mathcal{G}\left(x_{m_{1}}, x_{m_{2}}, \ldots, x_{m_{n-1}}, x\right)<\varepsilon^{\prime}$. In particular, if $m=m_{1}=m_{2}=\cdots=m_{n-1} \geq m_{0}$, then, by item (3),

$$
\mathcal{G}\left(x_{m},[x]^{n-1}\right) \leq(n-1) \mathcal{G}\left(x,\left[x_{m}\right]^{n-1}\right)<(n-1) \varepsilon^{\prime}=\varepsilon .
$$

Therefore, $\lim _{m \rightarrow \infty} \mathcal{G}\left(x_{m},[x]^{n-1}\right)=0$. 
Conversely, suppose that $\lim _{m \rightarrow \infty} \mathcal{G}\left(x_{m},[x]^{n-1}\right)=0$ and fix $\varepsilon>0$ arbitrary. Using $\varepsilon /(n-$ 1) $>0$, let $m_{0} \in \mathbb{N}$ be such that if $m \geq m_{0}$, then $\mathcal{G}\left(x_{m},[x]^{n-1}\right)<\varepsilon /(n-1)$. Therefore, by item (4) with $a=x$, if $m_{1}, m_{2}, \ldots, m_{n-1} \geq m_{0}$, we have

$$
\mathcal{G}\left(x_{m_{1}}, x_{m_{2}}, \ldots, x_{m_{n-1}}, x\right) \leq \sum_{i=1}^{n-1} \mathcal{G}\left(x_{m_{i}},[x]^{n-1}\right)<\sum_{i=1}^{n-1} \frac{\varepsilon}{n-1}=\varepsilon
$$

(6) Suppose that $\left\{x_{m}\right\}$ is $\mathcal{G}$-Cauchy and let $\varepsilon>0$. There is $m_{0} \in \mathbb{N}$ such that if $m_{1}, m_{2}$, $\ldots, m_{n-1}, m_{n} \geq m_{0}$, then $\mathcal{G}\left(x_{m_{1}}, x_{m_{2}}, \ldots, x_{m_{n-1}}, x_{m_{n}}\right)<\varepsilon$. Let $m, m^{\prime} \geq m_{0}$. Hence $\mathcal{G}\left(x_{m}\right.$, $\left.\left[x_{m^{\prime}}\right]^{n-1}\right)<\varepsilon$ and $\mathcal{G}\left(x_{m^{\prime}},\left[x_{m}\right]^{n-1}\right)<\varepsilon$ and we deduce that $d_{\mathcal{G}}\left(x_{m}, x_{m^{\prime}}\right)=\max \left(\mathcal{G}\left(x_{m},\left[x_{m^{\prime}}\right]^{n-1}\right)\right.$, $\left.\mathcal{G}\left(x_{m^{\prime}},\left[x_{m}\right]^{n-1}\right)\right)<\varepsilon$. Therefore, $\left\{x_{m}\right\}$ is $d_{\mathcal{G}}$-Cauchy.

Conversely, suppose that $\left\{x_{m}\right\}$ is $d_{\mathcal{G}}$-Cauchy and let $\varepsilon>0$. Given $\varepsilon / n>0$, there is $m_{0} \in \mathbb{N}$ such that if $m, m^{\prime} \geq m_{0}$, then $d_{\mathcal{G}}\left(x_{m}, x_{m^{\prime}}\right)<\varepsilon / n$. Therefore, if $m_{1}, m_{2}, \ldots, m_{n-1}, m_{n} \geq m_{0}$, item (4) ensures us that

$$
\mathcal{G}\left(x_{m_{1}}, x_{m_{2}}, \ldots, x_{m_{n-1}}, x_{m_{n}}\right) \leq \sum_{i=1}^{n} \mathcal{G}\left(x_{m_{i}},\left[x_{m_{0}}\right]^{n-1}\right) \leq \sum_{i=1}^{n} d_{\mathcal{G}}\left(x_{m_{i}}, x_{m_{0}}\right)<\sum_{i=1}^{n} \frac{\varepsilon}{n}=\varepsilon
$$

Hence, $\left\{x_{m}\right\}$ is $\mathcal{G}$-Cauchy.

Corollary 18 A sequence $\left\{x_{m}\right\}$ on a $G_{n}^{*}$-metric space $(X, \mathcal{G})$ is not $\mathcal{G}$-Cauchy if, and only if, there exist a positive $\varepsilon_{0}>0$ and two subsequences $\left\{x_{n\{k\}}\right\}_{k \geq 1}$ and $\left\{x_{m(k)}\right\}_{k \geq 1}$ of $\left\{x_{m}\right\}$ such that $k \leq n(k)<m(k)<n(k+1)$,

$$
\mathcal{G}\left(x_{m(k)},\left[x_{n(k)}\right]^{n-1}\right)>\varepsilon_{0} \quad \text { and } \quad \mathcal{G}\left(x_{m(k)-1},\left[x_{n(k)}\right]^{n-1}\right) \leq \varepsilon_{0} \quad \text { for all } k \in \mathbb{N} \text {. }
$$

To prove our main results, the following refinement of Corollary 18 plays a key role.

Lemma 19 Suppose that a sequence $\left\{x_{m}\right\}_{m \in \mathbb{N}}$ in a $G_{n}^{*}$-metric space $(X, \mathcal{G})$ is not $\mathcal{G}$-Cauchy and verifies $\left\{\mathcal{G}\left(x_{m+1},\left[x_{m}\right]^{n-1}\right)\right\}_{m \in \mathbb{N}} \rightarrow 0$. Then there exist $\varepsilon_{0}>0$ and two subsequences $\left\{x_{m(k)}\right\}_{k \in \mathbb{N}}$ and $\left\{x_{n(k)}\right\}_{k \in \mathbb{N}}$ such that, for all $k \in \mathbb{N}$,

$$
k \leq n(k)<m(k)<n(k+1), \quad \mathcal{G}\left(x_{m(k)},\left[x_{n(k)}\right]^{n-1}\right)>\varepsilon_{0} \quad \text { and } \quad \mathcal{G}\left(x_{m(k)-1},\left[x_{n(k)}\right]^{n-1}\right) \leq \varepsilon_{0} .
$$

Furthermore, for all $p_{1}, p_{2}, \ldots, p_{n} \geq 0$, we have

$$
\lim _{k \rightarrow \infty} \mathcal{G}\left(x_{n(k)+p_{1}}, x_{n(k)+p_{2}}, x_{n(k)+p_{3}}, \ldots, x_{n(k)+p_{n-1}}, x_{m(k)+p_{n}}\right)=\varepsilon_{0} .
$$

Moreover,

$$
\lim _{k \rightarrow \infty} \mathcal{G}\left(x_{n(k)-1}, x_{n(k)}, x_{n(k)+1}, x_{n(k)+2}, \ldots, x_{n(k)+n-3}, x_{m(k)-1}\right)=\varepsilon_{0} .
$$

In particular,

$$
\lim _{k \rightarrow \infty} \mathcal{G}\left(x_{m(k)},\left[x_{n(k)}\right]^{n-1}\right)=\varepsilon_{0} .
$$


Proof First part is Corollary 18. Now suppose that $\left\{x_{m}\right\}_{m \in \mathbb{N}}$ verifies

$$
\left\{\mathcal{G}\left(x_{m+1},\left[x_{m}\right]^{n-1}\right)\right\}_{m \in \mathbb{N}} \rightarrow 0 .
$$

By item (4) of Lemma 17, we have $0 \leq \mathcal{G}\left(x_{m},\left[x_{m+1}\right]^{n-1}\right) \leq(n-1) \mathcal{G}\left(x_{m+1},\left[x_{m}\right]^{n-1}\right)$ for all $m$, so

$$
\left\{\mathcal{G}\left(x_{m},\left[x_{m+1}\right]^{n-1}\right)\right\}_{m \in \mathbb{N}} \rightarrow 0 .
$$

Notice that, using $\left(\mathrm{A}_{4}\right)$,

$$
\begin{aligned}
\varepsilon_{0} & <\mathcal{G}\left(x_{m(k)},\left[x_{n(k)}\right]^{n-1}\right) \leq \mathcal{G}\left(x_{m(k)},\left[x_{m(k)-1}\right]^{n-1}\right)+\mathcal{G}\left(x_{m(k)-1},\left[x_{n(k)}\right]^{n-1}\right) \\
& \leq \mathcal{G}\left(x_{m(k)},\left[x_{m(k)-1}\right]^{n-1}\right)+\varepsilon_{0} .
\end{aligned}
$$

Using equation (3) and taking the limit as $k \rightarrow \infty$ we deduce that

$$
\lim _{k \rightarrow \infty} \mathcal{G}\left(x_{m(k)},\left[x_{n(k)}\right]^{n-1}\right)=\varepsilon_{0}
$$

Next we show, by induction, that for all $p_{1} \geq 0$,

$$
\lim _{k \rightarrow \infty} \mathcal{G}\left(x_{n(k)+p_{1}},\left[x_{n(k)}\right]^{n-2}, x_{m(k)}\right)=\varepsilon_{0} .
$$

If $p_{1}=0$, the claim holds by equation (5). Suppose that equation (6) holds for some $p_{1} \geq 0$; we will prove it for $p_{1}+1$. On the one hand,

$$
\mathcal{G}\left(x_{n(k)+p_{1}+1},\left[x_{n(k)}\right]^{n-2}, x_{m(k)}\right) \leq \mathcal{G}\left(x_{n(k)+p_{1}+1},\left[x_{n(k)+p_{1}}\right]^{n-1}\right)+\mathcal{G}\left(x_{n(k)+p_{1}},\left[x_{n(k)}\right]^{n-2}, x_{m(k)}\right),
$$

and, on the other hand,

$$
\mathcal{G}\left(x_{n(k)+p_{1}},\left[x_{n(k)}\right]^{n-2}, x_{m(k)}\right) \leq \mathcal{G}\left(x_{n(k)+p_{1}},\left[x_{n(k)+p_{1}+1}\right]^{n-1}\right)+\mathcal{G}\left(x_{n(k)+p_{1}+1},\left[x_{n(k)}\right]^{n-2}, x_{m(k)}\right) .
$$

Joining the two inequalities, for all $k \in \mathbb{N}$,

$$
\begin{aligned}
\mathcal{G} & \left(x_{n(k)+p_{1}},\left[x_{n(k)}\right]^{n-2}, x_{m(k)}\right)-\mathcal{G}\left(x_{n(k)+p_{1}},\left[x_{n(k)+p_{1}+1}\right]^{n-1}\right) \\
& \leq \mathcal{G}\left(x_{n(k)+p_{1}+1},\left[x_{n(k)}\right]^{n-2}, x_{m(k)}\right) \\
& \leq \mathcal{G}\left(x_{n(k)+p_{1}+1},\left[x_{n(k)+p_{1}}\right]^{n-1}\right)+\mathcal{G}\left(x_{n(k)+p_{1}},\left[x_{n(k)}\right]^{n-2}, x_{m(k)}\right)
\end{aligned}
$$

taking the limit as $k \rightarrow \infty$, and applying equations (3) and (4), we deduce that

$$
\varepsilon_{0}-0 \leq \lim _{k \rightarrow \infty} \mathcal{G}\left(x_{n(k)+p_{1}+1},\left[x_{n(k)}\right]^{n-2}, x_{m(k)}\right) \leq 0+\varepsilon_{0}
$$

that is, equation (6) holds for $p_{1}+1$. This completes the induction and equation (6) is valid for any $p_{1} \geq 0$. Next we prove that, for all $p_{1}, p_{2} \geq 0$,

$$
\lim _{k \rightarrow \infty} \mathcal{G}\left(x_{n(k)+p_{1}}, x_{n(k)+p_{2}},\left[x_{n(k)}\right]^{n-3}, x_{m(k)}\right)=\varepsilon_{0} .
$$


Fix $p_{1} \geq 0$ arbitrarily. If $p_{2}=0$, equation (7) is true by equation (6). Suppose that equation

(7) is true for some $p_{2} \geq 0$ and we will prove it for $p_{2}+1$. Indeed, on the one hand,

$$
\begin{aligned}
\mathcal{G} & \left(x_{n(k)+p_{1}}, x_{n(k)+p_{2}+1},\left[x_{n(k)}\right]^{n-3}, x_{m(k)}\right) \\
& =\mathcal{G}\left(x_{n(k)+p_{2}+1}, x_{n(k)+p_{1}},\left[x_{n(k)}\right]^{n-3}, x_{m(k)}\right) \\
& \leq \mathcal{G}\left(x_{n(k)+p_{2}+1},\left[x_{n(k)+p_{2}}\right]^{n-1}\right)+\mathcal{G}\left(x_{n(k)+p_{2}}, x_{n(k)+p_{1}},\left[x_{n(k)}\right]^{n-3}, x_{m(k)}\right),
\end{aligned}
$$

and, on the other hand,

$$
\begin{aligned}
& \mathcal{G}\left(x_{n(k)+p_{2}}, x_{n(k)+p_{1}},\left[x_{n(k)}\right]^{n-3}, x_{m(k)}\right) \\
& \quad \leq \mathcal{G}\left(x_{n(k)+p_{2}},\left[x_{n(k)+p_{2}+1}\right]^{n-1}\right)+\mathcal{G}\left(x_{n(k)+p_{2}+1}, x_{n(k)+p_{1}},\left[x_{n(k)}\right]^{n-3}, x_{m(k)}\right) .
\end{aligned}
$$

Combining the two inequalities, for all $k \in \mathbb{N}$,

$$
\begin{aligned}
\mathcal{G} & \left(x_{n(k)+p_{2}}, x_{n(k)+p_{1}},\left[x_{n(k)}\right]^{n-3}, x_{m(k)}\right)-\mathcal{G}\left(x_{n(k)+p_{2}},\left[x_{n(k)+p_{2}+1}\right]^{n-1}\right) \\
& \leq \mathcal{G}\left(x_{n(k)+p_{2}+1}, x_{n(k)+p_{1}},\left[x_{n(k)}\right]^{n-3}, x_{m(k)}\right) \\
& \leq \mathcal{G}\left(x_{n(k)+p_{2}+1},\left[x_{n(k)+p_{2}}\right]^{n-1}\right)+\mathcal{G}\left(x_{n(k)+p_{2}}, x_{n(k)+p_{1}},\left[x_{n(k)}\right]^{n-3}, x_{m(k)}\right) .
\end{aligned}
$$

Taking the limit as $k \rightarrow \infty$ and applying equations (3) and (4), we deduce that

$$
\varepsilon_{0}-0 \leq \lim _{k \rightarrow \infty} \mathcal{G}\left(x_{n(k)+p_{2}+1}, x_{n(k)+p_{1}},\left[x_{n(k)}\right]^{n-3}, x_{m(k)}\right) \leq 0+\varepsilon_{0}
$$

that is, equation (7) also holds for $p_{2}+1$. This completes the second induction. Repeating this reasoning in all arguments, we conclude that equation (2) holds. Exactly the same argument lets us prove that

$$
\lim _{k \rightarrow \infty} \mathcal{G}\left(x_{n(k)-1}, x_{n(k)}, x_{n(k)+1}, x_{n(k)+2}, \ldots, x_{n(k)+n-3}, x_{m(k)-1}\right)=\varepsilon_{0} .
$$

\section{Product of $G_{n}^{*}$-metric spaces}

In [11], the authors proved that, in general, the product space of $G$-metric spaces is not a $G$ metric space (unless the factors are symmetric, that is, that they can be reduced to metric spaces). Later, Roldán and Karapinar [20] introduced the concept of $G^{*}$-metric spaces, in which the axiom $\left(G_{3}\right)$ is omitted. Then they succeeded in proving that the product of $G^{*}$-metric spaces is also a $G^{*}$-metric space. This is the case of $G_{n}^{*}$-metric spaces.

Theorem 20 Let $\left\{\left(X_{i}, \mathcal{G}_{i}\right)\right\}_{i=1}^{N}$ be a family of $G^{*}$-metric spaces, consider the product space $\mathbb{X}=X_{1} \times X_{2} \times \cdots \times X_{N}$ and define $\mathcal{G}_{n}^{\max }$ and $\mathcal{G}_{n}^{\text {sum }}$ on $\mathbb{X}^{n}$ by

$$
\begin{aligned}
& \mathcal{G}_{n}^{\max }\left(\mathrm{Z}_{1}, \mathrm{Z}_{2}, \ldots, \mathrm{Z}_{n}\right)=\max _{1 \leq i \leq N} \mathcal{G}_{i}\left(z_{1}^{i}, z_{2}^{i}, \ldots, z_{n}^{i}\right) \text { and } \\
& G_{n}^{\text {sum }}\left(\mathrm{Z}_{1}, \mathrm{Z}_{2}, \ldots, \mathrm{Z}_{n}\right)=\sum_{i=1}^{N} \mathcal{G}_{i}\left(z_{i}^{1}, z_{i}^{2}, \ldots, z_{i}^{n}\right)
\end{aligned}
$$

for all $\mathrm{Z}_{1}=\left(z_{1}^{1}, z_{1}^{2}, \ldots, z_{1}^{N}\right), \mathrm{Z}_{2}=\left(z_{2}^{1}, z_{2}^{2}, \ldots, z_{2}^{N}\right), \ldots, \mathrm{Z}_{n}=\left(z_{n}^{1}, z_{n}^{2}, \ldots, z_{n}^{N}\right) \in \mathbb{X}$. Then the following statements hold. 
(1) $\mathcal{G}_{n}^{\max }$ and $\mathcal{G}_{n}^{\text {sum }}$ are $G_{n}^{*}$-metrics on $\mathbb{X}$. Also they are equivalent since $\mathcal{G}_{n}^{\max } \leq \mathcal{G}_{n}^{\text {sum }} \leq N \mathcal{G}_{n}^{\max }$.

(2) If $\mathrm{Z}_{m}=\left(z_{m}^{1}, z_{m}^{2}, \ldots, z_{m}^{N}\right) \in \mathbb{X}$ for all $m$ and $\mathrm{W}=\left(w^{1}, w^{2}, \ldots, w^{N}\right) \in \mathbb{X}$, then $\left\{Z_{m}\right\}$ $\mathcal{G}_{n}^{\max }$-converges (respectively, $\mathcal{G}_{n}^{\text {sum }}$-converges) to $\mathrm{W}$ if, and only if, each $\left\{z_{m}^{i}\right\}$ $\mathcal{G}_{i}$-converges to $w^{i}$.

(3) $\left\{Z_{m}\right\}_{m \geq 0}$ is $\mathcal{G}_{n}^{\max }$-Cauchy if, and only if, each $\left\{z_{m}^{i}\right\}_{m \geq 0}$ is $\mathcal{G}_{i}$-Cauchy for all $i \in\{1,2, \ldots, n\}$.

(4) $\left(\mathbb{X}, \mathcal{G}_{n}^{\max }\right)$ (respectively, $\left.\left(\mathbb{X}, \mathcal{G}_{n}^{\text {sum }}\right)\right)$ is complete if, and only if, every $\left(X_{i}, \mathcal{G}_{i}\right)$ is complete.

Proof We only reason with $\mathcal{G}_{n}^{\max }$ since the other case is similar. Note that if $\mathrm{Z}_{i}=$ $\left(z_{i}^{1}, z_{i}^{2}, \ldots, z_{i}^{N}\right) \in \mathbb{X}=X_{1} \times X_{2} \times \cdots \times X_{N}$, then $z_{i}^{j} \in X_{j}$ for all $i \in\{1,2, \ldots, n\}$ and all $j \in\{1,2, \ldots, N\}$.

(1) We prove four axioms.

$\left(\mathrm{A}_{1}\right)$ If $\mathrm{Z}=\left(z^{1}, z^{2}, \ldots, z^{N}\right) \in \mathbb{X}$, then $\mathcal{G}_{n}^{\max }(\mathrm{Z}, \mathrm{Z}, \ldots, \mathrm{Z})=\max _{1 \leq i \leq N} \mathcal{G}_{i}\left(z^{i}, z^{i}, \ldots, z^{i}\right)=$ $\max _{1 \leq i \leq N} 0=0$.

$\left(\mathrm{A}_{2}\right)$ Suppose that $\mathrm{Z}_{1} \neq \mathrm{Z}_{2}$, that is, there is $j \in\{1,2, \ldots, N\}$ such that $z_{1}^{j} \neq z_{2}$. Then $\mathcal{G}_{j}\left(z_{1}^{j}, z_{2}^{j}, \ldots, z_{n}^{j}\right)>0$ and we deduce that

$$
\mathcal{G}_{n}^{\max }\left(Z_{1}, Z_{2}, \ldots, Z_{n}\right)=\max _{1 \leq i \leq N} \mathcal{G}_{i}\left(z_{1}^{i}, z_{2}^{i}, \ldots, z_{n}^{i}\right) \geq \mathcal{G}_{j}\left(z_{1}^{j}, z_{2}^{j}, \ldots, z_{n}^{j}\right)>0
$$

$\left(\mathrm{A}_{3}\right)$ If $\sigma: \Lambda_{n} \rightarrow \Lambda_{n}$ is a permutation, then

$$
\begin{aligned}
\mathcal{G}_{n}^{\max }\left(\mathrm{Z}_{\sigma(1)}, \mathrm{Z}_{\sigma(2)}, \ldots, \mathrm{Z}_{\sigma(n)}\right) & =\max _{1 \leq i \leq N} \mathcal{G}_{i}\left(z_{\sigma(1)}^{i}, z_{\sigma(2)}^{i}, \ldots, z_{\sigma(n)}^{i}\right) \\
& =\max _{1 \leq i \leq N} \mathcal{G}_{i}\left(z_{1}^{i}, z_{2}^{i}, \ldots, z_{n}^{i}\right)=\mathcal{G}_{n}^{\max }\left(\mathrm{Z}_{1}, \mathrm{Z}_{2}, \ldots, \mathrm{Z}_{n}\right) .
\end{aligned}
$$

$\left(\mathrm{A}_{4}\right)$ Let $\mathrm{W}=\left(w^{1}, w^{2}, \ldots, w^{N}\right)$. Then

$$
\begin{aligned}
\mathcal{G}_{n}^{\max }\left(\mathrm{Z}_{1}, \mathrm{Z}_{2}, \ldots, \mathrm{Z}_{n}\right) & =\max _{1 \leq i \leq N} \mathcal{G}_{i}\left(z_{1}^{i}, z_{2}^{i}, \ldots, z_{n}^{i}\right) \\
& \leq \max _{1 \leq i \leq N}\left[\mathcal{G}_{i}\left(z_{1}^{i}, w^{i}, w^{i}, \ldots, w^{i}\right)+\mathcal{G}_{i}\left(w^{i}, z_{2}^{i}, z_{3}^{i}, \ldots, z_{n}^{i}\right)\right] \\
& \leq \max _{1 \leq i \leq N} \mathcal{G}_{i}\left(z_{1}^{i}, w^{i}, w^{i}, \ldots, w^{i}\right)+\max _{1 \leq i \leq N} \mathcal{G}_{i}\left(w^{i}, z_{2}^{i}, z_{3}^{i}, \ldots, z_{n}^{i}\right) \\
& =\mathcal{G}_{n}^{\max }\left(\mathrm{Z}_{1}, \mathrm{~W}, \mathrm{~W}, \ldots, \mathrm{W}\right)+\mathcal{G}_{n}^{\max }\left(\mathrm{W}, \mathrm{Z}_{2}, \mathrm{Z}_{3}, \ldots, \mathrm{Z}_{n}\right) .
\end{aligned}
$$

(2) It follows from Proposition 1, item (5) of Lemma 17, and the fact that

$$
\mathcal{G}_{n}^{\max }\left(\mathrm{Z}_{m}, \mathrm{~W}, \mathrm{~W}, \ldots, \mathrm{W}\right)=\max _{1 \leq i \leq N} \mathcal{G}_{i}\left(z_{m}^{i}, w^{i}, w^{i}, \ldots, w^{i}\right)
$$

(3) It is the same reasoning as taking into account that

$$
\mathcal{G}_{n}^{\max }\left(Z_{m_{1}}, \mathrm{Z}_{m_{2}}, \mathrm{Z}_{m_{3}}, \ldots, \mathrm{Z}_{m_{n}}\right)=\max _{1 \leq i \leq N} \mathcal{G}_{i}\left(z_{m_{1}}^{i}, z_{m_{2}}^{i}, z_{m_{3}}^{i}, \ldots, z_{m_{n}}^{i}\right)
$$

(4) Assume that $\left(X_{i}, \mathcal{G}_{i}\right)$ is complete for all $i \in\{1,2, \ldots, n\}$ and let $\left\{Z_{m}\right\}_{m \geq 0}$ be a $\mathcal{G}_{n}^{\max }$ Cauchy sequence in $\mathbb{X}$. By item (3), each sequence $\left\{z_{m}^{i}\right\}_{m \geq 0}$ is $\mathcal{G}_{i}$-Cauchy for all $i \in$ 
$\{1,2, \ldots, n\}$. Since $\left(X_{i}, \mathcal{G}_{i}\right)$ is complete, there is $w^{i} \in X$ such that $\left\{z_{m}^{i}\right\} \stackrel{\mathcal{G}_{i}}{\rightarrow} w^{i}$, for all $i \in$ $\{1,2, \ldots, n\}$. If $\mathrm{W}=\left(w^{1}, w^{2}, \ldots, w^{N}\right) \in \mathbb{X}$, item (2) guarantees that $\left\{Z_{m}\right\} \stackrel{\mathcal{G}_{n}^{\max }}{\rightarrow} \mathrm{W}$. Therefore, $\left(\mathbb{X}, \mathcal{G}_{n}^{\max }\right)$ is complete. The converse is similar.

\section{Some fixed point results on $G_{n}^{*}$-metric spaces}

The following result is a natural extension of Theorem 26 in [20] and of Theorem 3.1 in [22]. We highlight two facts: on the one hand, if $n \geq 3$, the following result cannot be reduced to metric spaces since the role of $x$ and $y$ is not symmetric; on the other hand, it cannot be reduced to $G$-metric spaces (three arguments) because repeating some arguments does not yield G-metric spaces (see Remark 11 and also Remark 2 in [22]).

Theorem 21 Let $(X, \preccurlyeq)$ be a preordered set endowed with a $G_{n}^{*}$-metric $\mathcal{G}$ and let $T: X \rightarrow X$ be a given mapping. Suppose that the following conditions hold:

(a) $(X, \mathcal{G})$ is complete.

(b) $T$ is non-decreasing (w.r.t. $\preccurlyeq)$.

(c) Either $T$ is $\mathcal{G}$-continuous or $(X, \mathcal{G}, \preccurlyeq)$ is regular-non-decreasing.

(d) There exists $x_{0} \in X$ such that $x_{0} \preccurlyeq T x_{0}$.

(e) There exist two mappings $\psi, \varphi \in \Psi$ such that, for all $x, y \in X$ with $x \preccurlyeq y$,

$$
\psi\left(\mathcal{G}\left(T x, T y, T^{2} x, T^{2} x, \ldots, T^{2} x\right)\right) \leq(\psi-\varphi)(\mathcal{G}(x, y, T x, T x, \ldots, T x))
$$

Then $T$ has a fixed point. Furthermore, if for all $z_{1}, z_{2} \in X$ fixed points of $T$ there exists $z \in X$ such that $z_{1} \preccurlyeq z$ and $z_{2} \preccurlyeq z$, we obtain uniqueness of the fixed point.

Proof Define $x_{m}=T^{m} x_{0}$ for all $m \geq 1$. Since $T$ is $\preccurlyeq$-non-decreasing, then $x_{m} \preccurlyeq x_{m+1}$ for all $m \geq 0$. Then

$$
\begin{aligned}
\psi\left(\mathcal{G}\left(x_{m+1},\left[x_{m+2}\right]^{n-1}\right)\right) & =\psi\left(\mathcal{G}\left(T x_{m}, T x_{m+1}, T^{2} x_{m}, T^{2} x_{m}, \ldots, T^{2} x_{m}\right)\right) \\
& \leq(\psi-\varphi)\left(\mathcal{G}\left(x_{m}, x_{m+1}, T x_{m}, T x_{m}, \ldots, T x_{m}\right)\right) \\
& =(\psi-\varphi)\left(\mathcal{G}\left(x_{m}, x_{m+1}, x_{m+1}, x_{m+1}, \ldots, x_{m+1}\right)\right) \\
& =(\psi-\varphi)\left(\mathcal{G}\left(x_{m},\left[x_{m+1}\right]^{n-1}\right)\right) .
\end{aligned}
$$

Applying Lemma $4,\left\{\mathcal{G}\left(x_{m},\left[x_{m+1}\right]^{n-1}\right)\right\} \rightarrow 0$. By item (4) of Lemma $17,0 \leq \mathcal{G}\left(x_{m+1}\right.$, $\left.\left[x_{m}\right]^{n-1}\right) \leq(n-1) \mathcal{G}\left(x_{m},\left[x_{m+1}\right]^{n-1}\right)$, so

$$
\left\{\mathcal{G}\left(x_{m},\left[x_{m+1}\right]^{n-1}\right)\right\} \rightarrow 0 \text { and } \quad\left\{\mathcal{G}\left(x_{m+1},\left[x_{m}\right]^{n-1}\right)\right\} \rightarrow 0
$$

Let us show that $\left\{x_{m}\right\}$ is $\mathcal{G}$-Cauchy. Reasoning by contradiction, if $\left\{x_{m}\right\}$ is not $\mathcal{G}$-Cauchy, by Lemma 19 , there exist $\varepsilon_{0}>0$ and two subsequences $\left\{x_{n(k)}\right\}$ and $\left\{x_{m(k)}\right\}$ verifying $k \leq$ $n(k)<m(k)$,

$$
\begin{aligned}
& \mathcal{G}\left(x_{m(k)}, x_{n(k)},\left[x_{n(k)+1}\right]^{n-2}\right)>\varepsilon_{0} \text { and } \\
& \mathcal{G}\left(x_{m(k)-1}, x_{n(k)},\left[x_{n(k)+1}\right]^{n-2}\right) \leq \varepsilon_{0} \text { for all } k \geq 1 .
\end{aligned}
$$


Therefore, by (8),

$$
\begin{aligned}
0 & <\psi\left(\varepsilon_{0}\right) \leq \psi\left(\mathcal{G}\left(x_{m(k)}, x_{n(k)},\left[x_{n(k)+1}\right]^{n-2}\right)\right) \\
& =\psi\left(\mathcal{G}\left(x_{n(k)}, x_{m(k)},\left[x_{n(k)+1}\right]^{n-2}\right)\right) \\
& =\psi\left(\mathcal{G}\left(T x_{n(k)-1}, T x_{m(k)-1},\left[T^{2} x_{n(k)-1}\right]^{n-2}\right)\right) \\
& \leq(\psi-\varphi)\left(\mathcal{G}\left(x_{n(k)-1}, x_{m(k)-1},\left[T x_{n(k)-1}\right]^{n-2}\right)\right) \\
& =\psi\left(\mathcal{G}\left(x_{n(k)-1}, x_{m(k)-1},\left[x_{n(k)}\right]^{n-2}\right)\right)-\varphi\left(\mathcal{G}\left(x_{n(k)-1}, x_{m(k)-1},\left[x_{n(k)}\right]^{n-2}\right)\right) .
\end{aligned}
$$

Consider the sequence of non-negative real numbers $\left\{\mathcal{G}\left(x_{n(k)-1}, x_{m(k)-1},\left[x_{n(k)}\right]^{n-2}\right)\right\}$. If this sequence has a subsequence converging to zero, then we can take the limit in equation (10) using this subsequence and we would deduce $0<\psi\left(\varepsilon_{0}\right) \leq 0$, which is impossible. Then $\left\{\mathcal{G}\left(x_{n(k)-1}, x_{m(k)-1},\left[x_{n(k)}\right]^{n-2}\right)\right\}$ cannot have a subsequence converging to zero. This means that there exist $\delta>0$ and $k_{0} \in \mathbb{N}$ such that

$$
\mathcal{G}\left(x_{n(k)-1}, x_{m(k)-1},\left[x_{n(k)}\right]^{n-2}\right) \geq \delta \quad \text { for all } k \geq k_{0} .
$$

Since $\varphi$ is non-decreasing, $-\varphi\left(\mathcal{G}\left(x_{n(k)-1}, x_{m(k)-1},\left[x_{n(k)}\right]^{n-2}\right)\right) \leq-\varphi(\delta)<0$. We also notice that, using $\left(\mathrm{A}_{4}\right)$,

$$
\begin{aligned}
\delta \leq & \mathcal{G}\left(x_{n(k)-1}, x_{m(k)-1},\left[x_{n(k)}\right]^{n-2}\right)=\mathcal{G}\left(x_{m(k)-1},\left[x_{n(k)}\right]^{n-2}, x_{n(k)-1}\right) \\
\leq & \mathcal{G}\left(x_{m(k)-1},\left[x_{n(k)+1}\right]^{n-1}\right)+\mathcal{G}\left(x_{n(k)+1},\left[x_{n(k)}\right]^{n-2}, x_{n(k)-1}\right) \\
= & \mathcal{G}\left(x_{n(k)+1},\left[x_{n(k)+1}\right]^{n-2}, x_{m(k)-1}\right)+\mathcal{G}\left(x_{n(k)+1},\left[x_{n(k)}\right]^{n-2}, x_{n(k)-1}\right) \\
\leq & \left(\mathcal{G}\left(x_{n(k)+1},\left[x_{n(k)}\right]^{n-1}\right)+\mathcal{G}\left(x_{n(k)},\left[x_{n(k)+1}\right]^{n-2}, x_{m(k)-1}\right)\right) \\
& +\left(\mathcal{G}\left(x_{n(k)+1},\left[x_{n(k)}\right]^{n-1}\right)+\mathcal{G}\left(x_{n(k)},\left[x_{n(k)}\right]^{n-2}, x_{n(k)-1}\right)\right) \\
\leq & \mathcal{G}\left(x_{n(k)+1},\left[x_{n(k)}\right]^{n-1}\right)+\varepsilon_{0}+\mathcal{G}\left(x_{n(k)+1},\left[x_{n(k)}\right]^{n-1}\right)+\mathcal{G}\left(x_{n(k)-1},\left[x_{n(k)}\right]^{n-1}\right) .
\end{aligned}
$$

By equations (10), (11), and (12), and taking into account that $\psi$ is non-decreasing, it follows that, for all $k \geq k_{0}$,

$$
\begin{aligned}
0< & \psi\left(\varepsilon_{0}\right) \leq \psi\left(\mathcal{G}\left(x_{n(k)-1}, x_{m(k)-1},\left[x_{n(k)}\right]^{n-2}\right)\right)-\varphi\left(\mathcal{G}\left(x_{n(k)-1}, x_{m(k)-1},\left[x_{n(k)}\right]^{n-2}\right)\right) \\
\leq & \psi\left(\mathcal{G}\left(x_{n(k)-1}, x_{m(k)-1},\left[x_{n(k)}\right]^{n-2}\right)\right)-\varphi(\delta) \\
\leq & \psi\left(\mathcal{G}\left(x_{n(k)+1},\left[x_{n(k)}\right]^{n-1}\right)+\varepsilon_{0}+\mathcal{G}\left(x_{n(k)+1},\left[x_{n(k)}\right]^{n-1}\right)\right. \\
& \left.+\mathcal{G}\left(x_{n(k)-1},\left[x_{n(k)}\right]^{n-1}\right)\right)-\varphi(\delta) .
\end{aligned}
$$

Using equation (9), the fact that $\psi$ is continuous and taking the limit when $k \rightarrow \infty$ in equation (13), we deduce that $0<\psi\left(\varepsilon_{0}\right) \leq \psi\left(\varepsilon_{0}\right)-\varphi(\delta)$, which is impossible since $\delta>0$ and $\varphi(\delta)>0$. This contradiction shows us that $\left\{x_{m}\right\}$ is a $\mathcal{G}$-Cauchy sequence. Since $(X, \mathcal{G})$ is complete, there exists $z_{0} \in X$ such that $\left\{x_{m}\right\} \stackrel{\mathcal{G}}{\rightarrow} z_{0}$.

Now suppose that $T$ is $\mathcal{G}$-continuous. Then $\left\{x_{m+1}\right\}=\left\{T x_{m}\right\} \stackrel{\mathcal{G}}{\rightarrow} T z_{0}$. By the unicity of the limit, $T z_{0}=z_{0}$, and $z_{0}$ is a fixed point of $T$. 
On the other case, suppose that $(X, \mathcal{G}, \preccurlyeq)$ is regular. Since $\left\{x_{m}\right\} \stackrel{\mathcal{G}}{\rightarrow} z_{0}$ and $\left\{x_{m}\right\}$ is monotone non-decreasing (w.r.t. $\preccurlyeq$ ), it follows that $x_{m} \preccurlyeq z_{0}$ for all $m$. Hence

$$
\begin{aligned}
\psi\left(\mathcal{G}\left(x_{m+1}, T z_{0},\left[x_{m+2}\right]^{n-2}\right)\right) & =\psi\left(\mathcal{G}\left(T x_{m}, T z_{0},\left[T^{2} x_{m}\right]^{n-2}\right)\right) \\
& \leq(\psi-\varphi)\left(\mathcal{G}\left(x_{m}, z_{0},\left[T x_{m}\right]^{n-2}\right)\right) \\
& =(\psi-\varphi)\left(\mathcal{G}\left(x_{m},\left[x_{m+1}\right]^{n-2}, z_{0}\right)\right) .
\end{aligned}
$$

Since $\left\{x_{m}\right\} \stackrel{\mathcal{G}}{\rightarrow} z_{0}$, then $\left\{\mathcal{G}\left(x_{m},\left[x_{m+1}\right]^{n-2}, z_{0}\right)\right\} \rightarrow 0$. Taking the limit when $k \rightarrow \infty$ we deduce that

$$
\left\{\psi\left(\mathcal{G}\left(x_{m+1}, T z_{0},\left[x_{m+2}\right]^{n-2}\right)\right)\right\} \rightarrow 0 .
$$

By Lemma $3,\left\{\mathcal{G}\left(x_{m+1}, T z_{0},\left[x_{m+2}\right]^{n-2}\right)\right\} \rightarrow 0$, so $\left\{x_{m}\right\} \stackrel{\mathcal{G}}{\rightarrow} T z_{0}$ and we also conclude that $z_{0}$ is a fixed point of $T$.

To prove the uniqueness, let $z_{1}, z_{2} \in X$ two fixed points of $T$. By hypothesis, there exists $z \in X$ such that $z_{1} \preccurlyeq z$ and $z_{2} \preccurlyeq z$. Let us show that $\left\{T^{m} z\right\} \stackrel{\mathcal{G}}{\rightarrow} z_{1}$. Indeed,

$$
\begin{aligned}
\psi\left(\mathcal{G}\left(T^{m+1} z,\left[z_{1}\right]^{n-1}\right)\right) & =\psi\left(\mathcal{G}\left(T z_{1}, T T^{m} z,\left[T^{2} z_{1}\right]^{n-2}\right)\right) \\
& \leq(\psi-\varphi)\left(\mathcal{G}\left(z_{1}, T^{m} z,\left[T z_{1}\right]^{n-2}\right)\right) \\
& =(\psi-\varphi)\left(\mathcal{G}\left(T^{m} z,\left[z_{1}\right]^{n-1}\right)\right) .
\end{aligned}
$$

By Lemma 4 , we deduce $\left\{\mathcal{G}\left(T^{m} z,\left[z_{1}\right]^{n-1}\right)\right\} \rightarrow 0$, that is, $\left\{T^{m} z\right\} \stackrel{\mathcal{G}}{\rightarrow} z_{1}$. The same reasoning proves that $\left\{T^{m} z\right\} \stackrel{\mathcal{G}}{\rightarrow} z_{2}$, so $z_{1}=z_{2}$.

If we particularize the previous result to the case in which $n=3$, we obtain the following consequence.

Corollary 22 (Roldán and Karapınar [20], Theorem 26) Let $(X, \preccurlyeq)$ be a preordered set endowed with $a G^{*}$-metric $G$ and $T: X \rightarrow X$ be a given mapping. Suppose that the following conditions hold:

(a) $(X, G)$ is complete.

(b) $T$ is non-decreasing (w.r.t. $\preccurlyeq)$.

(c) Either $T$ is $G$-continuous or $(X, G, \preccurlyeq)$ is regular-non-decreasing.

(d) There exists $x_{0} \in X$ such that $x_{0} \preccurlyeq T x_{0}$.

(e) There exist two mappings $\psi, \varphi \in \Psi$ such that, for all $x, y \in X$ with $x \preccurlyeq y$,

$$
\psi\left(G\left(T x, T y, T^{2} x\right)\right) \leq \psi(G(x, y, T x))-\varphi(G(x, y, T x))
$$

Then $T$ has a fixed point. Furthermore, if for all $z_{1}, z_{2} \in X$ fixed points of $T$ there exists $z \in X$ such that $z_{1} \preccurlyeq z$ and $z_{2} \preccurlyeq z$, we obtain uniqueness of the fixed point.

Taking $\psi(t)=t$ for all $t \geq 0$ in the previous theorem, we deduce the following result.

Corollary 23 Let $(X, \mathcal{G})$ be a complete $G_{n}^{*}$-metric space endowed with a preorder $\preccurlyeq$ and let $T: X \rightarrow X$ be $a \preccurlyeq$-non-decreasing mapping such that there exists $\varphi \in \Psi$ verifying, for 
all $x, y \in X$ with $x \preccurlyeq y$,

$$
\mathcal{G}\left(T x, T y, T^{2} x, T^{2} x, \ldots, T^{2} x\right) \leq \mathcal{G}(x, y, T x, T x, \ldots, T x)-\varphi(\mathcal{G}(x, y, T x, T x, \ldots, T x)) .
$$

Assume that $T$ is $\mathcal{G}$-continuous or $(X, \mathcal{G}, \preccurlyeq)$ is regular. Then $T$ has a fixed point provided that there is $x_{0} \in X$ such that $x_{0} \preccurlyeq T x_{0}$.

In addition to this, taking $\varphi(t)=(1-k) t$ for all $t \geq 0$ in the previous corollary, we deduce the following result.

Corollary 24 Let $(X, \mathcal{G})$ be a complete $G_{n}^{*}$-metric space endowed with a preorder $\preccurlyeq$ and let $T: X \rightarrow X$ be $a \preccurlyeq$-non-decreasing mapping such that there exists $k \in[0,1)$ verifying

$$
\mathcal{G}\left(T x, T y, T^{2} x, T^{2} x, \ldots, T^{2} x\right) \leq k \mathcal{G}(x, y, T x, T x, \ldots, T x) \quad \text { for all } x, y \in X \text { with } x \preccurlyeq y \text {. }
$$

Assume that $T$ is $\mathcal{G}$-continuous or $(X, \mathcal{G}, \preccurlyeq)$ is regular. Then $T$ has a fixed point provided that there is $x_{0} \in X$ such that $x_{0} \preccurlyeq T x_{0}$.

\section{Some fixed point results on $G_{n}$-metric spaces}

The following result is a variation of Theorem 21 in the setting of $G_{n}$-metric spaces.

Theorem 25 Let $(X, \preccurlyeq)$ be a preordered set endowed with a $G_{n}$-metric $\mathcal{G}$ and let $T: X \rightarrow X$ be a given mapping. Suppose that the following conditions hold:

(a) $(X, \mathcal{G})$ is complete.

(b) $T$ is non-decreasing (w.r.t. $\preccurlyeq)$.

(c) Either $T$ is $\mathcal{G}$-continuous.

(d) There exists $x_{0} \in X$ such that $x_{0} \preccurlyeq T x_{0}$.

(e) There exist two mappings $\psi \in \Psi$ and $\varphi \in \Phi$ such that, for all $x, y \in X$ with $x \preccurlyeq y$,

$$
\psi\left(\mathcal{G}\left(T x, T y, T^{2} x, T^{3} x, \ldots, T^{n-1} x\right)\right) \leq(\psi-\varphi)\left(\mathcal{G}\left(x, y, T x, T^{2} x, \ldots, T^{n-2} x\right)\right) .
$$

Then $T$ has a fixed point. Furthermore, if for all $z_{1}, z_{2} \in X$ fixed points of $T$ there exists $\omega \in X$ such that $z_{1} \preccurlyeq \omega$ and $z_{2} \preccurlyeq \omega$, we obtain uniqueness of the fixed point.

Proof Define $x_{m}=T^{m} x_{0}$ for all $m \geq 1$. If there exists some $m_{0} \in \mathbb{N}$ such that $x_{m_{0}}=x_{m_{0}+1}$, then $x_{m_{0}}$ is a fixed point of $T$. On the contrary, suppose that

$$
x_{m} \neq x_{m+1} \quad \text { for all } m \in \mathbb{N} \text {. }
$$

Since $T$ is $\preccurlyeq$-non-decreasing and $x_{0} \preccurlyeq T x_{0}=x_{1}$, then $x_{m} \preccurlyeq x_{m+1}$ for all $m \geq 0$. Therefore, taking $x=x_{m}$ and $y=x_{m+n-1}$ in equation (14), we have

$$
\begin{aligned}
\psi & \left(\mathcal{G}\left(x_{m+1}, x_{m+2}, x_{m+3}, \ldots, x_{m+n-1}, x_{m+n}\right)\right) \\
& =\psi\left(\mathcal{G}\left(T x_{m}, T^{2} x_{m}, T^{3} x_{m}, \ldots, T^{n-1} x_{m}, T x_{m+n-1}\right)\right) \\
& =\psi\left(\mathcal{G}\left(T x_{m}, T x_{m+n-1}, T^{2} x_{m}, T^{3} x_{m}, \ldots, T^{n-1} x_{m}\right)\right) \\
& \leq(\psi-\varphi)\left(\mathcal{G}\left(x_{m}, x_{m+n-1}, T x_{m}, T^{2} x_{m}, \ldots, T^{n-2} x_{m}\right)\right) \\
& =(\psi-\varphi)\left(\mathcal{G}\left(x_{m}, x_{m+1}, x_{m+2}, \ldots, x_{m+n-1}\right)\right) .
\end{aligned}
$$


Applying Lemma $4,\left\{\mathcal{G}\left(x_{m}, x_{m+1}, x_{m+2}, \ldots, x_{m+n-1}\right)\right\}_{m \geq 0} \rightarrow 0$. Taking into account equation (15) and $\left(\mathrm{A}_{5}\right)$,

$$
\begin{aligned}
\mathcal{G}\left(x_{m+1},\left[x_{m}\right]^{n-1}\right) & =\mathcal{G}\left(x_{m}, x_{m}, x_{m}, \ldots, x_{m}, x_{m+1}\right) \\
& \leq \mathcal{G}\left(x_{m}, x_{m+1}, x_{m+2}, \ldots, x_{m+n-1}\right) \quad \text { for all } m \geq 0 .
\end{aligned}
$$

Therefore,

$$
\left\{\mathcal{G}\left(x_{m+1},\left[x_{m}\right]^{n-1}\right)\right\}_{m \geq 0} \rightarrow 0 \text {. }
$$

Let us show that $\left\{x_{m}\right\}$ is $\mathcal{G}$-Cauchy. Reasoning by contradiction, if $\left\{x_{m}\right\}$ is not $\mathcal{G}$-Cauchy, by Lemma 19 , there exist $\varepsilon_{0}>0$ and two subsequences $\left\{x_{n(k)}\right\}$ and $\left\{x_{m(k)}\right\}$ verifying $k \leq n(k)<$ $m(k)<n(k+1)$,

$$
\begin{aligned}
& \mathcal{G}\left(x_{m(k)},\left[x_{n(k)}\right]^{n-2}\right)>\varepsilon_{0} \quad \text { and } \quad \mathcal{G}\left(x_{m(k)-1},\left[x_{n(k)}\right]^{n-1}\right) \leq \varepsilon_{0} \quad \text { for all } k \geq 1 \\
& \lim _{k \rightarrow \infty} \mathcal{G}\left(x_{n(k)+p_{1}}, x_{n(k)+p_{2}}, x_{n(k)+p_{3}}, \ldots, x_{n(k)+p_{n-1}, x_{m}(k)+p_{n}}\right)=\varepsilon_{0} \quad \text { for all } p_{1}, p_{2}, \ldots, p_{n} \geq 0 \\
& \lim _{k \rightarrow \infty} \mathcal{G}\left(x_{n(k)-1}, x_{n(k)}, x_{n(k)+1}, x_{n(k)+2}, \ldots, x_{n(k)+n-3}, x_{m(k)-1}\right)=\varepsilon_{0}
\end{aligned}
$$

In particular, taking appropriate values for $p_{1}, p_{2}, \ldots, p_{n} \geq 0$, we have

$$
\lim _{k \rightarrow \infty} \mathcal{G}\left(x_{n(k)}, x_{n(k)+1}, x_{n(k)+2}, \ldots, x_{n(k)+n-2}, x_{m(k)}\right)=\varepsilon_{0}
$$

From $x_{n(k)} \preccurlyeq x_{m(k)}$ and by equation (14),

$$
\begin{aligned}
\psi( & \left.\mathcal{G}\left(x_{n(k)}, x_{n(k)+1}, x_{n(k)+2}, \ldots, x_{n(k)+n-2}, x_{m(k)}\right)\right) \\
= & \psi\left(\mathcal{G}\left(x_{n(k)}, x_{m(k)}, x_{n(k)+1}, x_{n(k)+2}, \ldots, x_{n(k)+n-2}\right)\right) \\
= & \psi\left(\mathcal{G}\left(T x_{n(k)-1}, T x_{m(k)-1}, T^{2} x_{n(k)-1}, T^{3} x_{n(k)-1}, \ldots, T^{n-1} x_{n(k)-1}\right)\right) \\
\leq & (\psi-\varphi)\left(\mathcal{G}\left(x_{n(k)-1}, x_{m(k)-1}, T x_{n(k)-1}, T^{2} x_{n(k)-1}, \ldots, T^{n-2} x_{n(k)-1}\right)\right) \\
= & (\psi-\varphi)\left(\mathcal{G}\left(x_{n(k)-1}, x_{m(k)-1}, x_{n(k)}, x_{n(k)+1}, \ldots, x_{n(k)+n-3}\right)\right) \\
= & (\psi-\varphi)\left(\mathcal{G}\left(x_{n(k)-1}, x_{n(k)}, x_{n(k)+1}, \ldots, x_{n(k)+n-3}, x_{m(k)-1}\right)\right) \\
= & \psi\left(\mathcal{G}\left(x_{n(k)-1}, x_{n(k)}, x_{n(k)+1}, \ldots, x_{n(k)+n-3}, x_{m(k)-1}\right)\right) \\
& -\varphi\left(\mathcal{G}\left(x_{n(k)-1}, x_{n(k)}, x_{n(k)+1}, \ldots, x_{n(k)+n-3}, x_{m(k)-1}\right)\right) \\
\leq & \psi\left(\mathcal{G}\left(x_{n(k)-1}, x_{n(k)}, x_{n(k)+1}, \ldots, x_{n(k)+n-3}, x_{m(k)-1}\right)\right)
\end{aligned}
$$

for all $k \geq 0$. Using that $\psi$ is a continuous mapping, and equations (16) and (17), we deduce that the sequence

$$
\left\{\varphi\left(\mathcal{G}\left(x_{n(k)-1}, x_{n(k)}, x_{n(k)+1}, \ldots, x_{n(k)+n-3}, x_{m(k)-1}\right)\right)\right\}_{k \in \mathbb{N}}
$$

has a finite limit and, more precisely,

$$
\psi\left(\varepsilon_{0}\right) \leq \psi\left(\varepsilon_{0}\right)-\lim _{k \rightarrow \infty} \varphi\left(\mathcal{G}\left(x_{n(k)-1}, x_{n(k)}, x_{n(k)+1}, \ldots, x_{n(k)+n-3}, x_{m(k)-1}\right)\right) \leq \psi\left(\varepsilon_{0}\right)
$$


Hence

$$
\lim _{k \rightarrow \infty} \varphi\left(\mathcal{G}\left(x_{n(k)-1}, x_{n(k)}, x_{n(k)+1}, \ldots, x_{n(k)+n-3}, x_{m(k)-1}\right)\right)=0 .
$$

It follows from equation (1) that

$$
\varepsilon_{0}=\lim _{k \rightarrow \infty} \mathcal{G}\left(x_{n(k)-1}, x_{n(k)}, x_{n(k)+1}, \ldots, x_{n(k)+n-3}, x_{m(k)-1}\right)=0
$$

which contradicts that $\varepsilon_{0}>0$. This contradiction shows us that $\left\{x_{m}\right\}$ is a $\mathcal{G}$-Cauchy sequence. Since $(X, \mathcal{G})$ is complete, there exists $z_{0} \in X$ such that $\left\{x_{m}\right\} \stackrel{\mathcal{G}}{\rightarrow} z_{0}$. Furthermore, since $T$ is $\mathcal{G}$-continuous, then $\left\{x_{m+1}\right\}=\left\{T x_{m}\right\} \stackrel{\mathcal{G}}{\rightarrow} T z_{0}$. By the unicity of the limit, $T z_{0}=z_{0}$, and $z_{0}$ is a fixed point of $T$.

To prove the uniqueness, let $z_{1}, z_{2} \in X$ two fixed points of $T$. By hypothesis, there exists $\omega_{0} \in X$ such that $z_{1} \preccurlyeq \omega_{0}$ and $z_{2} \preccurlyeq \omega_{0}$. Let us show that $\left\{\omega_{m}=T^{m} \omega_{0}\right\} \stackrel{\mathcal{G}}{\rightarrow} z_{1}$. On the one hand, since $T$ is non-decreasing, $z_{1}=T^{m} z_{1} \preccurlyeq T^{m} \omega_{0}=\omega_{m}$. On the other hand, for all $m \geq 0$,

$$
\begin{aligned}
\psi\left(\mathcal{G}\left(\omega_{m+1},\left[z_{1}\right]^{n-1}\right)\right) & =\psi\left(\mathcal{G}\left(T z_{1}, T \omega_{m}, T^{2} z_{1}, T^{3} z_{1}, \ldots, T^{n-1} z_{1}\right)\right) \\
& \leq(\psi-\varphi)\left(\mathcal{G}\left(z_{1}, \omega_{m}, T z_{1}, T^{2} z_{1}, \ldots, T^{n-2} z_{1}\right)\right) \\
& =(\psi-\varphi)\left(\mathcal{G}\left(\omega_{m},\left[z_{1}\right]^{n-1}\right)\right) .
\end{aligned}
$$

By Lemma 4 , we deduce $\left\{\mathcal{G}\left(\omega_{m},\left[z_{1}\right]^{n-1}\right)\right\} \rightarrow 0$, that is, by item (5) of Lemma $17,\left\{\omega_{m}=\right.$ $\left.T^{m} \omega_{0}\right\} \stackrel{\mathcal{G}}{\rightarrow} z_{1}$. The same reasoning proves that $\left\{T^{m} \omega_{0}\right\} \stackrel{\mathcal{G}}{\rightarrow} z_{2}$, so $z_{1}=z_{2}$.

In the following result, we suppose that $\varphi \in \Psi$, and we analyze the case in which $T$ is not necessarily continuous.

Theorem 26 Let $(X, \preccurlyeq)$ be a preordered set endowed with a $G_{n}$-metric $\mathcal{G}$ and let $T: X \rightarrow X$ be a given mapping. Suppose that the following conditions hold:

(a) $(X, \mathcal{G})$ is complete.

(b) $T$ is non-decreasing (w.r.t. $\preccurlyeq)$.

(c) Either $T$ is $\mathcal{G}$-continuous or $(X, \mathcal{G}, \preccurlyeq)$ is regular.

(d) There exists $x_{0} \in X$ such that $x_{0} \preccurlyeq T x_{0}$.

(e) There exist two mappings $\psi, \varphi \in \Psi$ such that, for all $x, y \in X$ with $x \preccurlyeq y$,

$$
\psi\left(\mathcal{G}\left(T x, T y, T^{2} x, T^{3} x, \ldots, T^{n-1} x\right)\right) \leq(\psi-\varphi)\left(\mathcal{G}\left(x, y, T x, T^{2} x, \ldots, T^{n-2} x\right)\right)
$$

Then $T$ has a fixed point. Furthermore, if for all $z_{1}, z_{2} \in X$ fixed points of $T$ there exists $z \in X$ such that $z_{1} \preccurlyeq z$ and $z_{2} \preccurlyeq z$, we obtain uniqueness of the fixed point.

Proof By Remark 6, $\varphi \in \Psi \subseteq \Phi$, so this result holds when $T$ is continuous. Now suppose that $(X, \mathcal{G}, \preccurlyeq)$ is regular. Repeating the previous proof, we know that $\left\{x_{m}=T^{m} x_{0}\right\} \stackrel{\mathcal{G}}{\rightarrow} z_{0}$. Since $\left\{x_{m}\right\}$ is $\preccurlyeq$-non-decreasing and $(X, \mathcal{G}, \preccurlyeq)$ is regular, it follows that $x_{m} \preccurlyeq z_{0}$ for all $m$. Hence

$$
\begin{aligned}
\psi & \left(\mathcal{G}\left(x_{m+1}, x_{m+2}, x_{m+3}, \ldots, x_{m+n-1}, T z_{0}\right)\right) \\
& =\psi\left(\mathcal{G}\left(T x_{m}, T z_{0}, T^{2} x_{m}, T^{3} x_{m}, \ldots, T^{n-1} x_{m}\right)\right)
\end{aligned}
$$




$$
\begin{aligned}
& \leq(\psi-\varphi)\left(\mathcal{G}\left(x_{m}, z_{0}, T x_{m}, T^{2} x_{m}, \ldots, T^{n-2} x_{m}\right)\right) \\
& =(\psi-\varphi)\left(\mathcal{G}\left(x_{m}, x_{m+1}, x_{m+2}, \ldots, x_{m+n-2}, z_{0}\right)\right) .
\end{aligned}
$$

As $\left\{x_{m}\right\} \stackrel{\mathcal{G}}{\rightarrow} z_{0}$, we know that $\left\{\mathcal{G}\left(x_{m}, x_{m+1}, x_{m+2}, \ldots, x_{m+n-2}, z_{0}\right)\right\}_{m \geq 0} \rightarrow 0$. Since $\psi$ and $\varphi$ are continuous, then $\left\{(\psi-\varphi)\left(\mathcal{G}\left(x_{m}, x_{m+1}, x_{m+2}, \ldots, x_{m+n-2}, z_{0}\right)\right)\right\}_{m \geq 0} \rightarrow 0$, so $\left\{\psi\left(\mathcal{G}\left(x_{m+1}, x_{m+2}\right.\right.\right.$, $\left.\left.\left.x_{m+3}, \ldots, x_{m+n-1}, T z_{0}\right)\right)\right\}_{m \geq 0} \rightarrow 0$. By Lemma 3 ,

$$
\left\{\mathcal{G}\left(x_{m+1}, x_{m+2}, x_{m+3}, \ldots, x_{m+n-1}, T z_{0}\right)\right\}_{m \geq 0} \rightarrow 0
$$

and this means that $\left\{x_{m+1}\right\} \stackrel{\mathcal{G}}{\rightarrow} T z_{0}$. The unicity of the limit leads us to conclude that $T z_{0}=z_{0}$.

Theorems 25 and 26 can be particularized to the case in which $\psi(t)=t$ for all $t \geq 0$ as follows.

Corollary 27 Let $(X, \mathcal{G})$ be a complete $G_{n}$-metric space endowed with a preorder $\preccurlyeq$ and let $T: X \rightarrow X$ be $a \preccurlyeq$-non-decreasing, $\mathcal{G}$-continuous mapping such that there exists $\varphi \in \Phi$ verifying, for all $x, y \in X$ with $x \preccurlyeq y$,

$$
\begin{aligned}
& \psi\left(\mathcal{G}\left(T x, T y, T^{2} x, T^{3} x, \ldots, T^{n-1} x\right)\right) \\
& \quad \leq \mathcal{G}\left(x, y, T x, T^{2} x, \ldots, T^{n-2} x\right)-\varphi\left(\mathcal{G}\left(x, y, T x, T^{2} x, \ldots, T^{n-2} x\right)\right) .
\end{aligned}
$$

Then $T$ has a fixed point provided that there is $x_{0} \in X$ such that $x_{0} \preccurlyeq T x_{0}$.

Corollary 28 Let $(X, \mathcal{G})$ be a complete $G_{n}$-metric space endowed with a preorder $\preccurlyeq$ and let $T: X \rightarrow X$ be $a \preccurlyeq$-non-decreasing mapping such that there exists $\varphi \in \Psi$ verifying, for all $x, y \in X$ with $x \preccurlyeq y$,

$$
\begin{aligned}
& \mathcal{G}\left(T x, T y, T^{2} x, T^{3} x, \ldots, T^{n-1} x\right) \\
& \quad \leq \mathcal{G}\left(x, y, T x, T^{2} x, \ldots, T^{n-2} x\right)-\varphi\left(\mathcal{G}\left(x, y, T x, T^{2} x, \ldots, T^{n-2} x\right)\right) .
\end{aligned}
$$

Assume that $T$ is $\mathcal{G}$-continuous or $(X, \mathcal{G}, \preccurlyeq)$ is regular. Then $T$ has a fixed point provided that there is $x_{0} \in X$ such that $x_{0} \preccurlyeq T x_{0}$.

The following result is a particularization to the case in which $\varphi(t)=(1-k) t$ for all $t \geq 0$, where $k \in[0,1)$.

Corollary 29 Let $(X, \mathcal{G})$ be a complete $G_{n}$-metric space endowed with a preorder $\preccurlyeq$ and let $T: X \rightarrow X$ be $a \preccurlyeq$-non-decreasing mapping such that there exists $k \in[0,1)$ verifying, for all $x, y \in X$ with $x \preccurlyeq y$,

$$
\mathcal{G}\left(T x, T y, T^{2} x, T^{3} x, \ldots, T^{n-1} x\right) \leq k \mathcal{G}\left(x, y, T x, T^{2} x, \ldots, T^{n-2} x\right)
$$

Assume that $T$ is $\mathcal{G}$-continuous or $(X, \mathcal{G}, \preccurlyeq)$ is regular. Then $T$ has a fixed point provided that there is $x_{0} \in X$ such that $x_{0} \preccurlyeq T x_{0}$. 


\section{Consequences}

Similar (but easier) techniques permit us to prove the following result.

Theorem 30 Let $(X, \preccurlyeq)$ be a preordered set endowed with a $G_{n}^{*}$-metric $\mathcal{G}$ and let $T: X \rightarrow X$ be a given mapping. Suppose that the following conditions hold:

(a) $(X, \mathcal{G})$ is complete.

(b) $T$ is non-decreasing (w.r.t. $\preccurlyeq)$.

(c) Either $T$ is $\mathcal{G}$-continuous or $(X, \mathcal{G}, \preccurlyeq)$ is regular-non-decreasing.

(d) There exists $x_{0} \in X$ such that $x_{0} \preccurlyeq T x_{0}$.

(e) There exist two mappings $\psi, \varphi \in \Psi$ such that, for all $x_{1}, x_{2}, \ldots, x_{n} \in X$ with $x_{1} \preccurlyeq x_{2} \preccurlyeq x_{3} \preccurlyeq \cdots \preccurlyeq x_{n}$,

$$
\psi\left(\mathcal{G}\left(T x_{1}, T x_{2}, T x_{3}, \ldots, T x_{n}\right)\right) \leq(\psi-\varphi)\left(\mathcal{G}\left(x_{1}, x_{2}, x_{3}, \ldots, x_{n}\right)\right) .
$$

Then $T$ has a fixed point. Furthermore, if for all $z_{1}, z_{2} \in X$ fixed points of $T$ there exists $z \in X$ such that $z_{1} \preccurlyeq z$ and $z_{2} \preccurlyeq z$, we obtain uniqueness of the fixed point.

In particular, taking $Y=X \times X$, we can conclude the following result.

Corollary 31 (Choudhury and Maity [22], Theorem 3.1) Let $(X, \preccurlyeq)$ be a partially ordered set and $G$ be a G-metric on $X$ such that $(X, G)$ is a complete G-metric space. Let $F: X \times X \rightarrow$ $X$ be a continuous mapping having the mixed monotone property on $X$. Assume that there exists a $k \in[0,1)$ such that for $x, y, z, u, v, w \in X$, the following holds:

$$
G(F(x, y), F(u, v), F(w, z)) \leq \frac{k}{2}[G(x, u, w)+G(y, v, z)]
$$

for all $x \succcurlyeq u \succcurlyeq w$ and $y \preccurlyeq v \preccurlyeq z$ where either $u \neq w$ or $v \neq z$.

If there exist $x_{0}, y_{0} \in X$ such that $x_{0} \preccurlyeq F\left(x_{0}, y_{0}\right)$ and $y_{0} \succcurlyeq F\left(y_{0}, x_{0}\right)$, then $F$ has a coupled fixed point in $X$, that is, there exist $x, y \in X$ such that $x=F(x, y)$ and $y=F(y, x)$.

Competing interests

The authors declare that they have no competing interests.

Authors' contributions

All authors contributed equally and significantly in writing this paper. All authors read and approved the final manuscript.

\section{Author details}

'University of Jaén, Campus las Lagunillas s/n, Jaén, 23071, Spain. ²Department of Mathematics, Atilim University, Incek, Ankara, Turkey. ${ }^{3}$ Department of Mathematics, Faculty of Science, King Mongkut's University of Technology Thonburi (KMUTT), Bangkok, 10140, Thailand.

\section{Acknowledgements}

The first author has been partially supported by Junta de Andalucía by project FQM-268 of the Andalusian CICYE. The third author was supported by the Higher Education Research Promotion and National Research University Project of Thailand, Office of the Higher Education Commission (Under NRU-CSEC Project No. NRU56000508).

\footnotetext{
Endnote

a Notice that in this axiom, it is possible that $x=x_{3}$ or $y=x_{4}$.
} 


\section{References}

1. Gähler, S: 2-Metrische Räume und ihr topologische struktur. Math. Nachr. 26, 115-148 (1963)

2. Gähler, S: Zur geometric 2-metrische räume. Rev. Roum. Math. Pures Appl. XL, 664-669 (1966)

3. Dhage, BC: Generalized metric space and mapping with fixed point. Bull. Calcutta Math. Soc. 84, $329-336$ (1992)

4. Ha, KS, Cho, YJ, White, A: Strictly convex and strictly 2-convex 2-normed spaces. Math. Jpn. 33, 375-384 (1988)

5. Dhage, BC: Generalized metric space and topological structure I. An. Stiinț. Univ. 'Al.I. Cuza' laşi, Mat. 46, 3-24 (2000)

6. Dhage, BC: On generalized metric spaces and topological structure II. Pure Appl. Math. Sci. 40, 37-41 (1994)

7. Dhage, BC: On continuity of mappings in D-metric spaces. Bull. Calcutta Math. Soc. 86, 503-508 (1994)

8. Mustafa, Z, Sims, B: Some remarks concerning D-metric spaces. In: Proceedings of the International Conferences on Fixed Point Theorey and Applications, Valencia (Spain), July, pp. 189-198 (2003)

9. Naidu, SVR, Rao, KPR, Rao, S: On the concepts of balls in a D-metric space. Int. J. Math. Math. Sci. 1, 133-141 (2005)

10. Mustafa, Z: A new structure for generalized metric spaces with applications to fixed point theory. PhD thesis, The University of Newcastle, Australia (2005)

11. Mustafa, Z, Sims, B: A new approach to generalized metric spaces. J. Nonlinear Convex Anal. 7, $289-297$ (2006)

12. Mustafa, Z, Obiedat, $H$, Awawdeh, F: Some fixed point theorem for mapping on complete G-metric spaces. Fixed Point Theory Appl. 2008, Article ID 189870 (2008)

13. Mustafa, Z, Khandaqji, M, Shatanawi, W: Fixed point results on complete G-metric spaces. Studia Sci. Math. Hung. 48 , 304-319 (2011)

14. Mustafa, Z, Shatanawi, W, Bataineh, M: Existence of fixed point results in G-metric spaces. Int. J. Math. Math. Sci. 2009, Article ID 283028 (2009)

15. Shatanawi, W: Coupled fixed point theorems in generalized metric spaces. Hacet. J. Math. Stat. 40, 441-447 (2011)

16. Agarwal, RP, Karapınar, E: Remarks on some coupled fixed point theorems in G-metric spaces. Fixed Point Theory Appl. 2013, 2 (2013)

17. Alghamdi, MA, Karapınar, E: G- $\beta-\psi$ Contractive type mappings and related fixed point theorems. J. Inequal. Appl. 2013, 70 (2013)

18. Karapınar, E, Agarwal, RP: Further fixed point results on G-metric spaces. Fixed Point Theory Appl. 2013,154 (2013)

19. Karapınar, E, Kumam, P, Erhan, IM: Coupled fixed point theorems on partially ordered G-metric spaces. Fixed Point Theory Appl. 2012, 174 (2012)

20. Roldán, A, Karapınar, E: Some multidimensional fixed point theorems on partially preordered $G^{*}$-metric spaces under $(\psi, \varphi)$-contractivity conditions. Fixed Point Theory Appl. 2013, 158 (2013)

21. Ding, H-S, Karapınar, E: Meir-Keeler type contractions in partially ordered G-metric spaces. Fixed Point Theory Appl. 2013, 35 (2013)

22. Choudhury, BS, Maity, P: Coupled fixed point results in generalized metric spaces. Math. Comput. Model. 54, 73-79 (2011)

23. Roldán, A, Martínez-Moreno, J, Roldán, C: Multidimensional fixed point theorems in partially ordered complete metric spaces. J. Math. Anal. Appl. 396, 536-545 (2012)

24. Roldán, A, Martínez-Moreno, J, Roldán, C, Karapınar, E: Meir-Keeler type multidimensional fixed point theorems in partially ordered metric spaces. Abstr. Appl. Anal. 2013, Article ID 406026 (2013)

25. Mustafa, Z, Sims, B: Fixed point theorems for contractive mappings in complete G-metric spaces. Fixed Point Theory Appl. 2009, Article ID 917175 (2009)

26. Roldán, A, Martínez-Moreno, J, Roldán, C, Karapınar, E: Multidimensional fixed point theorems in partially ordered complete partial metric spaces under $(\psi, \varphi)$-contractivity conditions. Abstr. Appl. Anal. 2013, Article ID 634371 (2013)

27. Khan, MS, Swaleh, M, Sessa, S: Fixed point theorems by altering distances between the points. Bull. Aust. Math. Soc 30, 1-9 (1984)

28. Popescu, O: Fixed points for $(\psi, \phi)$-weak contractions. Appl. Math. Lett. 24(1), 1-4 (2011)

29. Moradi, S, Farajzadeh, A: On the fixed point of $(\psi-\varphi)$-weak and generalized $(\psi-\varphi)$-weak contraction mappings. Appl. Math. Lett. 25(10), 1257-1262 (2012)

10.1186/1687-1812-2014-13

Cite this article as: Roldán et al.: G-Metric spaces in any number of arguments and related fixed-point theorems. Fixed Point Theory and Applications 2014, 2014:13

\section{Submit your manuscript to a SpringerOpen ${ }^{\odot}$ journal and benefit from:}

- Convenient online submission

- Rigorous peer review

- Immediate publication on acceptance

- Open access: articles freely available online

- High visibility within the field

- Retaining the copyright to your article 